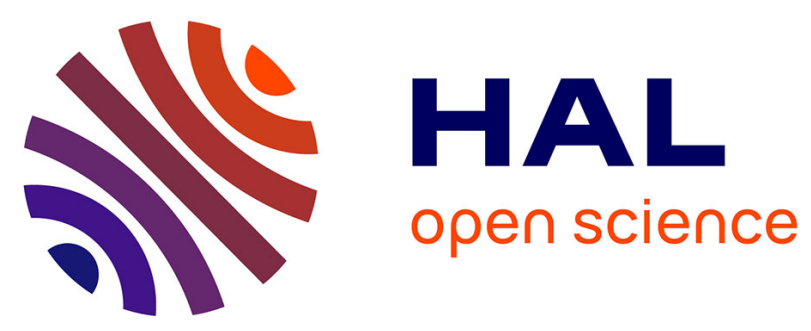

\title{
Inverse parametric linear/quadratic programming problem for continuous PWA functions defined on polyhedral partitions of polyhedra
}

\author{
Ngoc Anh Nguyen, Sorin Olaru, Pedro Rodriguez-Ayerbe
}

\section{To cite this version:}

Ngoc Anh Nguyen, Sorin Olaru, Pedro Rodriguez-Ayerbe. Inverse parametric linear/quadratic programming problem for continuous PWA functions defined on polyhedral partitions of polyhedra. 54th IEEE Conference on Decision and Control (CDC), Dec 2015, OSAKA, Japan. pp.5926-5931, 10.1109/CDC.2015.7403150 . hal-01334079

\section{HAL Id: hal-01334079}

https://hal-centralesupelec.archives-ouvertes.fr/hal-01334079

Submitted on 22 Jun 2016

HAL is a multi-disciplinary open access archive for the deposit and dissemination of scientific research documents, whether they are published or not. The documents may come from teaching and research institutions in France or abroad, or from public or private research centers.
L'archive ouverte pluridisciplinaire HAL, est destinée au dépôt et à la diffusion de documents scientifiques de niveau recherche, publiés ou non, émanant des établissements d'enseignement et de recherche français ou étrangers, des laboratoires publics ou privés. 


\title{
Inverse parametric linear/quadratic programming problem for continuous PWA functions defined on polyhedral partitions of polyhedra
}

\author{
N. A. Nguyen ${ }^{1}$, S. Olaru ${ }^{1}$, P. Rodriguez-Ayerbe ${ }^{1}$
}

\begin{abstract}
Constructive solution to inverse parametric linear/quadratic programming problems has recently been investigated and shown to be solvable via convex liftings [15], [14]. These results were stated and solved starting from polytopic partitions of a polytope in the parameter space. Therefore, the case of polyhedral partitions of unbounded polyhedra, was not handled by this method and deserves a complete characterization to address the general inverse optimality problem. This paper has as main objective to overcome the unboundedness limitation of the given polyhedral partition and to extend the constructive solution put forward in [14] for this omitted case.
\end{abstract}

\section{INTRODUCTION}

Inverse parametric convex programming was previously investigated in [1] where it was proved that every continuous nonlinear function can be obtained via parametric convex programming without a guideline for the construction of such convex programming problems. The first constructive results have recently been presented via different approaches in [7], [14], [15]. These developments focus on continuous piecewise affine functions, a particular but important class of nonlinear functions which receives great attention in control theory as attested by the references in different topics [3], [9], [22], [10], [18], [2], [16], [19], [4].

It is well known that optimal solution to a parametric linear/quadratic programming problem is a piecewise affine (PWA) function defined over a polyhedral partition. Note that the continuity of optimal solution to parametric linear programming problems, is not guaranteed in many cases. However, it is proved in [17] that a continuous optimal solution can always be selected. Conversely, inverse parametric linear/quadratic programming (IPL/QP) aims to recover an appropriate optimization problem, described by a constraint set and a cost function, having as the optimal solution a given continuous PWA function defined over a polyhedral partition.

This paper completes and generalizes the result in [14], [15] wherein IPL/QP problems are solved via convex liftings. In the respective works, this solution covers polytopic partitions (i.e. bounded polyhedral partitions) of the parameter space, since the convex lifting construction is based on continuity and convexity conditions on vertices of the polytopes composing the given polytopic partition. Therefore, if the given polyhedral partition contains unbounded polyhedra,

N. A. Nguyen, S. Olaru, P. Rodriguez-Ayerbe are with the Laboratory of Signals and Systems (L2S, UMR CNRS 8506), CentraleSupélec-CNRSUniversité Paris Sud, 3 rue Joliot Curie, Plateau de Moulon, 91192, Gif-sur-Yvette, France. Ngocanh.Nguyen, Sorin.Olaru, Pedro.Rodriguez-Ayerbe @supelec.fr this algorithm cannot be directly employed. This paper is motivated by this limitation to solve IPL/QP problems for more general class of continuous PWA functions, namely continuous PWA functions defined over polyhedral partitions of polyhedra. This result is useful to reduce implementation complexity of PWA control laws as shown in [12], [6].

\section{PROBLEM FORMULATION}

\section{A. Generalities and basic notions}

Throughout the paper, $\mathbb{R}, \mathbb{R}_{+}, \mathbb{R}_{>0}, \mathbb{N}, \mathbb{N}_{>0}$ denote the field of real numbers, the field of non-negative real numbers, the set of strictly positive real numbers, the set of nonnegative integers and the positive integer set, respectively. The following index set is also defined for ease of presentation with respect to a given $N \in \mathbb{N}_{>0}: \mathcal{I}_{N}=\left\{i \in \mathbb{N}_{>0} \mid\right.$ $i \leq N\}$.

Given a $d \in \mathbb{N}_{>0}$, by $1_{d}$, we denote a vector of dimension $d$ whose elements are equal to 1 ; i.e. $1_{d} \in \mathbb{R}^{d}$.

Given a finite set $\mathcal{S}:=\left\{s_{1}, \ldots, s_{L}\right\}$, then $\operatorname{Card}(\mathcal{S})$ denotes the cardinal number of the set $\mathcal{S}$, i.e. $\operatorname{Card}(\mathcal{S})=L \cdot \operatorname{conv}(\mathcal{S})$ denotes the convex hull of $\mathcal{S}$. Moreover, if $\mathcal{R}$ is a set of vectors, by cone $(\mathcal{R})$, we denote the cone which has elements of $\mathcal{R}$ as its extreme rays.

A polyhedron is defined as the intersection of a finite number of halfspaces. A polytope is defined as a bounded polyhedron. Given a full dimensional polyhedron $\mathcal{S} \subset \mathbb{R}^{d}$, then $\mathcal{V}(\mathcal{S})$ denotes the set of its vertices, and $\mathcal{R}(\mathcal{S})$ denotes the set of its extreme rays. We use $k$-face to denote a face of dimension $k$ of $\mathcal{S}$. A 0 -face is also called a vertex, an 1-face is alternatively called an edge, a $(d-1)$-face amounts to a facet. Furthermore, by $\operatorname{int}(\mathcal{S})$, we denote the interior of $\mathcal{S}$. If $\mathbb{S}$ is a vector space, and $\mathcal{S}$ is an arbitrary set, then $\operatorname{Proj}_{\mathbb{S}} \mathcal{S}$ represents the orthogonal projection of set $\mathcal{S}$ onto space $\mathbb{S}$. Also, we use $\operatorname{dim}(\mathcal{S})$ to denote the dimension of the affine hull of $\mathcal{S}$.

Finally, given two sets $P_{1}, P_{2} \subset \mathbb{R}^{d}$, by $P_{1} \oplus P_{2}$, we denote the Minkowski addition defined as follows:

$P_{1} \oplus P_{2}=\left\{y \in \mathbb{R}^{d} \mid \exists x_{1} \in P_{1}, x_{2} \in P_{2}\right.$ s.t. $\left.y=x_{1}+x_{2}\right\}$.

The following definitions are extended in comparison with their counterparts presented and employed in [15], [14], [12].

Definition 1: A collection of $N \in \mathbb{N}_{>0}$ full-dimensional polyhedra $\mathcal{X}_{i} \subset \mathbb{R}^{d}$ is called a polyhedral partition of a polyhedron $\mathcal{X}$ in $\mathbb{R}^{d}$ if:

1) $\mathcal{X}=\bigcup_{i \in \mathcal{I}_{N}} \mathcal{X}_{i}$

2) $\operatorname{int}\left(\mathcal{X}_{i}\right) \bigcap \operatorname{int}\left(\mathcal{X}_{j}\right)=\emptyset$ with $i \neq j,(i, j) \in \mathcal{I}_{N}^{2}$,

Also, $\left(\mathcal{X}_{i}, \mathcal{X}_{j}\right)$ are called neighbours if $(i, j) \in \mathcal{I}_{N}^{2}, i \neq j$ and $\operatorname{dim}\left(\mathcal{X}_{i} \cap \mathcal{X}_{j}\right)=d-1$. 
If set $\mathcal{X}$ is compact, then this partition is called polytopic partition.

The definition of a cell complex was presented by Grünbaum in [5]. For simplicity, a cell complex should be understood as a polyhedral partition whose facet-to-facet property is fulfilled; i.e. any pair of neighboring regions share a common facet.

In the remainder of this paper, a slight abuse of notation is used: $\mathcal{X}$ implies simultaneously a polyhedral partition/cell complex of a polyhedron and this polyhedron itself. Its meaning will be clarified from the context.

Definition 2: For a given polyhedral partition $\mathcal{X}=$ $\bigcup_{i \in \mathcal{I}_{N}} \mathcal{X}_{i} \subseteq \mathbb{R}^{d}$, a picewise affine lifting is described by a real-valued function $z: \mathcal{X} \rightarrow \mathbb{R}$ with:

$$
z(x)=a_{i}^{T} x+b_{i} \text { for any } x \in \mathcal{X}_{i},
$$

and $a_{i} \in \mathbb{R}^{d}, b_{i} \in \mathbb{R}, \forall i \in \mathcal{I}_{N}$.

Definition 3: Given a polyhedral partition $\mathcal{X}=\bigcup_{i \in \mathcal{I}_{N}} \mathcal{X}_{i}$ $\subseteq \mathbb{R}^{d}$, a piecewise affine lifting $z(x)=a_{i}^{T} x+b_{i} \forall x \in$ $\mathcal{X}_{i}$, is called convex piecewise affine lifting if the following conditions hold true:

- $z(x)$ is continuous over $\mathcal{X}$,

- for each $i \in \mathcal{I}_{N}, z(x)>a_{j}^{T} x+b_{j}$ for all $x \in \mathcal{X}_{i} \backslash \mathcal{X}_{j}$ and all $j \neq i, j \in \mathcal{I}_{N}$.

For ease of presentation, a convex piecewise affine lifting is called, throughout the rest of this paper, convex lifting. It is shown in [20], [13] that a polyhedral partition which admits a convex lifting, is a cell complex. Therefore, a convex lifting is always defined over a cell complex instead of a polyhedral partition.

For a given polyhedral partition, some conditions have to be fulfilled for the existence of a convex lifting. A summary of these conditions can be found in [20]. We extract here only the necessary notation in connection with the present developments. Interested readers can find details in [20], [15].

Given a polyhedron $C$ and its facet $F$, we use $n(F, C)$ to denote the inward unit normal vector at $F$ to $C$, meaning the unit vector normal to $F$ at $F$ and inward to $C$. The definition of stress is recalled here for completeness.

Definition 4: (Rybnikov [20]) A real-valued function $s(\cdot)$ defined on the $(d-1)$-faces of a cell complex $\mathcal{X} \subseteq \mathbb{R}^{d}$ is called a $d$-stress if at each internal $(d-2)$-face $F$ of $\mathcal{X}$ :

$$
\sum_{F \subset C} s(C) n(F, C)=0
$$

where this sum ranges over all $(d-1)$-faces in the star of $F$ (the $(d-1)$-faces such that $F$ is their common facet). The quantities $s(C)$ are the coefficients of $d$-stresses, and are called tension if the sign is strictly positive, and compression if the sign is strictly negative.

Such a $(d-2)$-face $F$ of cell complex $\mathcal{X}$ satisfying (2) is also called in equilibrium. Note also that a necessary and sufficient condition for such a cell complex $\mathcal{X}$ to be convexly liftable is the existence of a strictly positive $d$-stress defined over all $(d-1)$-faces of cell complex $\mathcal{X}$.

\section{B. Problem formulation}

The solution to inverse parametric linear/quadratic programming problems is shown in [15], [14] via convex liftings. This solution restricts the parameter space to a polytope and lets aside the important case of unbounded parameter space. This omission is tackled in this paper and the problem can be formally stated as follows:

Problem statement: Given a polyhedral partition of a polyhedron $\mathcal{X}=\bigcup_{i \in \mathcal{I}_{N}} \mathcal{X}_{i} \subseteq \mathbb{R}^{d_{x}}$, we use $f_{\text {pwa }}(\cdot)$ to denote a continuous PWA function: $f_{p w a}(x): \mathcal{X} \rightarrow \mathbb{R}^{d_{u}}$, defined over partition $\mathcal{X}$. The objective is to determine $J(x, z, u), H_{x}, H_{u}, H_{z}, K$ such that:

$$
\left\{\begin{aligned}
f_{p w a}(x)= & \operatorname{Proj}_{\mathbb{R}^{d_{u}}} \text { arg } \min _{\left[z u^{T}\right]^{T}} J(x, z, u), \\
\text { s.t: } \quad & H_{x} x+H_{z} z+H_{u} u \leq K .
\end{aligned}\right.
$$

Some assumptions need to be stated for guaranteeing the validity of the proposal.

Assumption 1: Polyhedral partition $\mathcal{X}$ is a cell complex.

Assumption 2: Polyhedral partition $\mathcal{X}$ is convexly liftable. As discussed before, a polyhedral partition, admitting a convex lifting, is a cell complex, therefore, if a given partition satisfies Assumption 2, then it also fulfills Assumption 1.

Note that Assumption 2 is not restrictive, since, as shown in [15], any polyhedral partition can be subdivided into a convexly liftable cell complex such that the internal boundaries are maintained. This inclusion can be easily extended to unbounded polyhedral partitions by following the same methodology of the proof (Theorem IV.2 in [15]).

\section{Constructive Solution}

It is shown in [15], [14] that constructive solution to such an inverse parametric linear/quadratic programming problem can be built upon convex lifting principle. However, the algorithm to construct convex liftings of the given cell complex put forward therein, is only applicable for cell complexes of polytopes. Therefore, for the cell complexes of polyhedra, this algorithm cannot be directly applicable. To adapt to unbounded parameter space, two intuitive approaches can be proposed:

- either to modify this algorithm subject to cell complexes of polyhedra,

- or to adjust the given cell complex of polyhedra in such a manner that this algorithm can be directly of use.

The latter approach will be of interest via a simply intermediate step in the sequel. More precisely, we restrict the given parameter space partition to an appropriate bounded region such that the convex liftability of this new partition and the initial one is treated in a similar manner. Accordingly, we propose here the use of a hyperbox large enough to contain in its interior all vertices of the initial partition. The existence of such a hyperbox is guaranteed by the fact that a polyhedral partition is the collection of a finite number of polyhedra, as a consequence the number of vertices is finite. By $B_{d_{x}}(\epsilon)$, we denote a hyperbox for a given scalar $\epsilon \in \mathbb{R}_{>0}$, defined as

$$
B_{d_{x}}(\epsilon):=\left\{x \in \mathbb{R}^{d_{x}} \mid\|x\|_{\infty} \leq \epsilon\right\} .
$$


The hyperbox used for our purpose needs to meet the following assumption:

Assumption 3: Given a polyhedral partition $\mathcal{X}=$ $\bigcup_{i \in \mathcal{I}_{N}} \mathcal{X}_{i} \subseteq \mathbb{R}^{d_{x}}$, the scalar $\epsilon$ is restricted to the values that ensure $x \in \operatorname{int}\left(B_{d_{x}}(\epsilon)\right)$ for every $x \in \bigcup_{i \in \mathcal{I}_{N}} \mathcal{V}\left(\mathcal{X}_{i}\right)$.

The following observation guarantees the correspondence of their convex liftability property.

Proposition 1: If a given partition $\mathcal{X}$ of a polyhedron satisfies Assumption 1, then partition $\mathcal{X}$ is convexly liftable if and only if there exists a hyperbox $B_{d_{x}}(\epsilon)$, satisfying Assumption 3 such that the partition $\mathcal{X} \cap B_{d_{x}}(\epsilon)=\bigcup_{i \in \mathcal{I}_{N}}\left(\mathcal{X}_{i} \cap\right.$ $\left.B_{d_{x}}(\epsilon)\right)$ is convexly liftable.

Proof: $\longrightarrow$ It is already known ([20], [15]) that the convex liftability of partition $\mathcal{X}$ leads to the existence of a strictly positive function $s(\cdot)$ defined over the $\left(d_{x}-1\right)$-faces of partition $\mathcal{X}$ such that every internal $\left(d_{x}-2\right)$-face is in equilibrium. Consider an internal $\left(d_{x}-2\right)$-face of partition $\mathcal{X}$ denoted by $F$, we write $\mathcal{F}_{d_{x}-1}(F)$ to denote the set of $\left(d_{x}-1\right)$-faces of partition $\mathcal{X}$, sharing a common facet $F$, then the following inclusion holds true:

$$
\sum_{C_{i} \in \mathcal{F}_{d_{x}-1}(F)} s\left(C_{i}\right) n\left(F, C_{i}\right)=0 .
$$

By the construction of $B_{d_{x}}(\epsilon)$, if $F$ is an internal $\left(d_{x}-\right.$ $2)$-face of partition $\mathcal{X}$, then $F \cap B_{d_{x}}(\epsilon)$ is also an internal $\left(d_{x}-2\right)$-face of partition $\mathcal{X} \cap B_{d_{x}}(\epsilon)$. Furthermore, $F \cap$ $B_{d_{x}}(\epsilon)$ also satisfies the following property:

$$
\sum_{C_{i} \in \mathcal{F}_{d_{x}-1}(F)} s\left(C_{i}\right) n\left(F \cap B_{d_{x}}(\epsilon), C_{i} \cap B_{d_{x}}(\epsilon)\right)=0,
$$

due to the fact that $n\left(F \cap B_{d_{x}}(\epsilon), C_{i} \cap B_{d_{x}}(\epsilon)\right)=n\left(F, C_{i}\right)$. Therefore any internal face $F \cap B_{d_{x}}(\epsilon)$ is in equilibrium with strictly positive $d_{x}$-stress: $s\left(C_{i} \cap B_{d_{x}}(\epsilon)\right)=s\left(C_{i}\right)>0$, meaning the cell complex $\mathcal{X} \cap B_{d_{x}}(\epsilon)$ is convexly liftable. $\longleftarrow$ The sufficient condition can be similarly proved.

The goal is to present a construction of convex liftings for the given cell complex $\mathcal{X}$ of a polyhedron, based on an intermediate polytopic partition $\mathcal{X} \cap B_{d_{x}}(\epsilon)$. The following observation shows this close relation.

Proposition 2: Given a cell complex $\mathcal{X}$ of a polyhedron satisfying Assumption 2 and a hyperbox $B_{d_{x}}(\epsilon)$ satisfying Assumption 3 , if $f: \mathcal{X} \cap B_{d_{x}}(\epsilon) \rightarrow \mathbb{R}$ i.e.

$$
f(x)=a_{i} x+b_{i} \text { for } x \in \mathcal{X}_{i} \cap B_{d_{x}}(\epsilon),
$$

is a convex lifting of the cell complex $\mathcal{X} \cap B_{d_{x}}(\epsilon)$, then the function $g: \mathcal{X} \rightarrow \mathbb{R}$, defined as follows:

$$
g(x)=a_{i} x+b_{i} \text { for } x \in \mathcal{X}_{i}
$$

is also a convex lifting of partition $\mathcal{X}$.

Proof: First, we observe that due to Assumption 3, the constraints characterized by $B_{d_{x}}(\epsilon)$ do not contribute to the division of the partition $\mathcal{X} \cap B_{d_{x}}(\epsilon)$.

From the hypothesis, $f(x)$ is a convex lifting of partition $\mathcal{X} \cap B_{d_{x}}(\epsilon)$, then it can be deduced from two neighboring regions $\left(\mathcal{X}_{i} \cap B_{d_{x}}(\epsilon), \mathcal{X}_{j} \cap B_{d_{x}}(\epsilon)\right)$ that:

$$
\begin{aligned}
& a_{i} x+b_{i}=a_{j} x+b_{j} \forall x \in \mathcal{X}_{i} \cap \mathcal{X}_{j} \cap B_{d_{x}}(\epsilon), \\
& a_{i} x+b_{i}>a_{j} x+b_{j} \forall x \in\left(\mathcal{X}_{i} \cap B_{d_{x}}(\epsilon)\right) \backslash\left(\mathcal{X}_{j} \cap B_{d_{x}}(\epsilon)\right) .
\end{aligned}
$$

Note also that the constraint

$$
a_{i} x+b_{i}=a_{j} x+b_{j}
$$

describes the set of points which lie on the hyperplane separating $\mathcal{X}_{i} \cap B_{d_{x}}(\epsilon)$ and $\mathcal{X}_{j} \cap B_{d_{x}}(\epsilon)$, then it also separates $\mathcal{X}_{i}$ and $\mathcal{X}_{j}$. Therefore, the following inclusions hold true:

$$
\begin{aligned}
& a_{i} x+b_{i}=a_{j} x+b_{j} \forall x \in \mathcal{X}_{i} \cap \mathcal{X}_{j}, \\
& a_{i} x+b_{i}>a_{j} x+b_{j} \forall x \in \mathcal{X}_{i} \backslash \mathcal{X}_{j} .
\end{aligned}
$$

The same argument holds true for all pairs of neighboring regions in partition $\mathcal{X} \cap B_{d_{x}}(\epsilon)$, leading to:

$$
g(x)=a_{i} x+b_{i}>a_{j} x+b_{j}, \forall x \in \mathcal{X}_{i} \backslash \mathcal{X}_{j}, \forall j \neq i
$$

In other words, as per Definition $3, g(x)$ is a convex lifting of the cell complex $\mathcal{X}$.

The above results are meaningful to construct a convex lifting for the cell complex $\mathcal{X}$ of a polyhedron, based on a bounded one $\mathcal{X} \cap B_{d_{x}}(\epsilon)$. The structure of the latter partition is compatible with the algorithms presented in [15].

As previously shown, the construction of a convex lifting for partition $\mathcal{X}$ of a polyhedron can be carried out via the restriction of $\mathcal{X}$ into a large enough hyperbox in the same dimensional space. A natural question arises how this hyperbox can be chosen. This choice must clearly satisfy Assumption 3, and can be found through Algorithm 1.

$$
\begin{aligned}
& \hline \text { Algorithm } 1 \text { Computation of a hyperbox } B_{d_{x}}(\epsilon) \\
& \text { Input: A cell complex } \mathcal{X} \text { of a polyhedron in } \mathbb{R}^{d_{x}} \text { and a scalar } \\
& \begin{array}{l}
\text { Output: } \epsilon \text {. } \\
\text { Out }
\end{array} \\
& \text { 1: } V=\bigcup_{i \in \mathcal{I}_{N}} \mathcal{V}\left(\mathcal{X}_{i}\right) \text {. } \\
& \text { 2: Solve the following problem } \\
& \quad \min _{\epsilon} \epsilon \\
& \text { s.t. } \epsilon \geq 0,-(\epsilon-a) 1_{d_{x}} \leq x \leq(\epsilon-a) 1_{d_{x}}, \forall x \in V .
\end{aligned}
$$

Note that such a constant $a>0$ needs to be inserted in the set of constraints for guaranteeing Assumption 3 i.e. all vertices $v \in \bigcup_{i \in \mathcal{I}_{N}} \mathcal{V}\left(\mathcal{X}_{i}\right)$ lie in the interior of $B_{d_{x}}(\epsilon)$. This constant can be freely chosen as long as it is strictly positive.

Based on these results, to state a solution to inverse parametric linear/quadratic programming problem via convex liftings for unbounded partition, we need to define some supplementary notation.

First, given a cell complex of a polyhedron $\mathcal{X}=$ $\bigcup_{i \in \mathcal{I}_{N}} \mathcal{X}_{i} \subseteq \mathbb{R}^{d_{x}}$ satisfying Assumption 2, a continuous PWA function $f_{\text {pwa }}(\cdot): \mathcal{X} \rightarrow \mathbb{R}^{d_{u}}$ is defined as

$$
f_{p w a}(x)=f_{i} x+g_{i} \text { for } x \in \mathcal{X}_{i}
$$


By $\ell(x)$, we denote a convex lifting of the cell complex $\mathcal{X}$ i.e. $\ell(x)=a_{i} x+b_{i}$ for $x \in \mathcal{X}_{i}$. Based on the above elements, the following sets are also defined:

$$
\begin{aligned}
& V_{x}=\bigcup_{i \in \mathcal{I}_{N}} \mathcal{V}\left(\mathcal{X}_{i}\right), R_{x}=\bigcup_{i \in \mathcal{I}_{N}} \mathcal{R}\left(\mathcal{X}_{i}\right), \\
& V_{\left[x^{T} z u^{T}\right]^{T}}=\left\{\left[x^{T} \ell(x) f_{p w a}^{T}(x)\right]^{T} \mid x \in V_{x}\right\},
\end{aligned}
$$

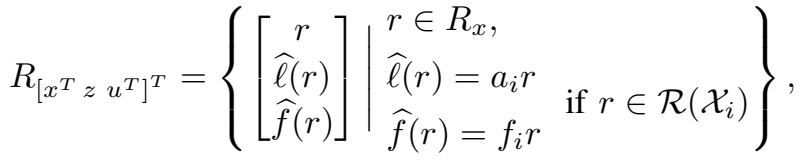

$$
\begin{aligned}
& \Pi_{v}=\operatorname{conv}\left(V_{\left[x^{T} z u^{T}\right]^{T}}\right), \Pi_{r}=\operatorname{cone}\left(R_{\left[x^{T} z u^{T}\right]^{T}}\right), \\
& \Pi=\Pi_{v} \oplus \Pi_{r} .
\end{aligned}
$$

With these notation, the following theorem presents a solution to IPL/QP problems via convex liftings for polyhedral partitions of a polyhedron.

Theorem 3.1: Given any continuous PWA function $f_{p w a}(x)$ (5) defined over a cell complex of a polyhedron satisfying Assumption 2 and the sets defined in (6), then $f_{p w a}(x)$ is the image via the orthogonal projection of the optimal solution to the following optimization problem:

$$
\min _{\left[z u^{T}\right]^{T}} z \quad \text { s.t. }\left[x^{T} z u^{T}\right]^{T} \in \Pi \text {. }
$$

Proof: Consider $x \in \mathcal{X}_{i}$, for a region $\mathcal{X}_{i}$ of the given partition $\mathcal{X}$, according to the Minkowski-Weyl theorem for polyhedra (Corollary 7.1b in [21]), $x$ can be described as follows:

$$
x=\sum_{v \in \mathcal{V}\left(\mathcal{X}_{i}\right)} \alpha(v) v+\sum_{r \in \mathcal{R}\left(\mathcal{X}_{i}\right)} \beta(r) r,
$$

where $\alpha(v), \beta(r) \in \mathbb{R}_{+}$and $\sum_{v \in \mathcal{V}\left(\mathcal{X}_{i}\right)} \alpha(v)=1$. As a consequence, the convex lifting at $x$, i.e. $\ell(x)$ can be described by:

$$
\begin{aligned}
\ell(x) & =a_{i} x+b_{i}=a_{i}\left(\sum_{v \in \mathcal{V}\left(\mathcal{X}_{i}\right)} \alpha(v) v+\sum_{r \in \mathcal{R}\left(\mathcal{X}_{i}\right)} \beta(r) r\right)+b_{i}, \\
& =\sum_{v \in \mathcal{V}\left(\mathcal{X}_{i}\right)} \alpha(v)\left(a_{i} v+b_{i}\right)+\sum_{r \in \mathcal{R}\left(\mathcal{X}_{i}\right)} \beta(r)\left(a_{i} r\right) .
\end{aligned}
$$

Similarly,

$$
\begin{aligned}
f_{p w a}(x) & =f_{i} x+g_{i} \\
& =f_{i}\left(\sum_{v \in \mathcal{V}\left(\mathcal{X}_{i}\right)} \alpha(v) v+\sum_{r \in \mathcal{R}\left(\mathcal{X}_{i}\right)} \beta(r) r\right)+g_{i}, \\
& =\sum_{v \in \mathcal{V}\left(\mathcal{X}_{i}\right)} \alpha(v)\left(f_{i} v+g_{i}\right)+\sum_{r \in \mathcal{R}\left(\mathcal{X}_{i}\right)} \beta(r)\left(f_{i} r\right) .
\end{aligned}
$$

It can be observed that if $r$ is an extreme ray of $\mathcal{X}_{i}$, then $\left[\begin{array}{ll}r^{T} & a_{i} r\end{array}\right]^{T}$ is also an extreme ray of the affinely equivalent polyhedron $\Pi_{\left[x^{T} z\right]^{T}}$ of cell complex $\mathcal{X}$ defined as follows:

$$
\left.\Pi_{\left[x^{T} z\right.}\right]^{T}=\operatorname{conv}\left(V_{\left[x^{T} z\right]^{T}}\right) \oplus \operatorname{cone}\left(R_{\left[x^{T} z\right]^{T}}\right),
$$

where

$$
\begin{aligned}
V_{\left[x^{T} z\right]^{T}} & =\left\{\left[\begin{array}{c}
x \\
\ell(x)
\end{array}\right] \mid x \in V_{x}\right\}, \\
R_{\left[x^{T} z\right]^{T}} & =\left\{\left[\begin{array}{c}
r \\
\widehat{\ell}(r)
\end{array}\right], \begin{array}{l}
r \in R_{x}, \\
\hat{\ell}(r)=a_{i} r \text { if } r \in \mathcal{R}\left(\mathcal{X}_{i}\right)
\end{array}\right\} .
\end{aligned}
$$

According to the definition of an affinely equivalent polyhedron, the optimal solution to the following problem:

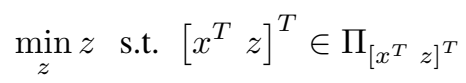

falls in the lower facets of $\Pi_{\left[x^{T} z\right]^{T}}$ described by the convex lifting $\ell(x)$ of partition $\mathcal{X}$. Now, consider a region $\mathcal{X}_{i}$, by $F_{\left[\begin{array}{ll}x^{T} & z\end{array}\right]^{T}}^{(i)}$ we denote the lower facet of $\left.\Pi_{\left[x^{T} z\right.} z\right]^{T}$ such that $\operatorname{Proj}_{\mathbb{R}^{d_{x}}} F_{\left[x^{T} z\right]^{T}}^{(i)}=\mathcal{X}_{i}$. According to Proposition 5.1 in [14], every augmented point in $V_{\left[x^{T} z u^{T}\right]^{T}}$ corresponds to the vertices of $\Pi_{v}$ after lifting onto $\mathbb{R}^{d_{x}+d_{u}+1}$. Therefore, by preserving lifting onto $\mathbb{R}^{d_{x}+d_{u}+1}$, we guarantee the existence

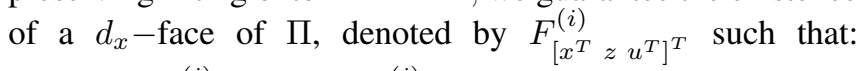

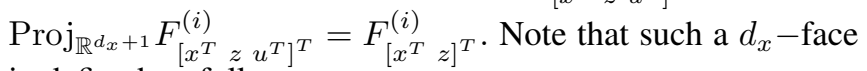
is defined as follows:

$$
\begin{aligned}
& F_{\left[x^{T} z u^{T}\right]^{T}}^{(i)}=F_{1}^{(i)} \oplus F_{2}^{(i)} \\
& F_{1}^{(i)}=\operatorname{conv}\left\{\left[v^{T} \ell(v) f_{p w a}^{T}(v)\right]^{T} \mid v \in \mathcal{V}\left(\mathcal{X}_{i}\right)\right\} \\
& F_{2}^{(i)}=\text { cone }\left\{\left[\begin{array}{c}
r \\
\widehat{\ell}(r) \\
\widehat{f}(r)
\end{array}\right] \mid r \in \mathcal{R}\left(\mathcal{X}_{i}\right), \begin{array}{c}
\widehat{\ell}(r)=a_{i} r \\
\widehat{f}(r)=f_{i} r
\end{array}\right\} .
\end{aligned}
$$

It can be observed that at a point $x \in \mathcal{X}_{i}$, the minimal value of $z$ can be obtained when $\left[x^{T} z u^{T}\right]^{T}$ lies in

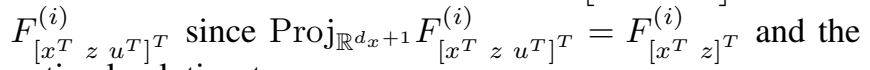
optimal solution to:

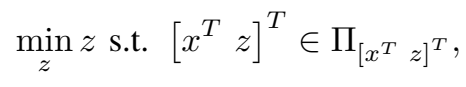

falls at the point $\left[x^{T} \ell(x)\right]^{T} \in F_{\left[x^{T} z\right]^{T}}^{(i)}$. Therefore, optimal solution to (7) at $x$ can be described by:

$$
\left[\begin{array}{c}
x \\
z^{*}(x) \\
u^{*}(x)
\end{array}\right]=\sum_{v \in \mathcal{V}\left(\mathcal{X}_{i}\right)} \alpha(v)\left[\begin{array}{c}
v \\
a_{i} v+b_{i} \\
f_{i} v+g_{i}
\end{array}\right]+\sum_{r \in \mathcal{R}\left(\mathcal{X}_{i}\right)} \beta(r)\left[\begin{array}{c}
r \\
a_{i} r \\
f_{i} r
\end{array}\right],
$$

where $\alpha(v), \beta(r) \in \mathbb{R}_{+}$and $\sum_{v \in \mathcal{V}\left(\mathcal{X}_{i}\right)} \alpha(v)=1$. It is clear that

$$
\left[\begin{array}{c}
z^{*}(x) \\
u^{*}(x)
\end{array}\right]=\left[\begin{array}{l}
a_{i} x+b_{i} \\
f_{i} x+g_{i}
\end{array}\right]=\left[\begin{array}{c}
\ell(x) \\
f_{p w a}(x)
\end{array}\right], \text { for } x \in \mathcal{X}_{i} .
$$

To complete the proof, we now need to show that the optimal solution to (7) is unique. In fact, at a point $x \in$ $\mathcal{X}_{i}$, suppose there exist two different $d_{x}$-faces denoted by $F_{1}, F_{2}$ to which optimal solutions to (7) belong. Let $\left[x^{T} z_{1}(x) u_{1}^{T}(x)\right]^{T} \in F_{1}$ and $\left[x^{T} z_{2}(x) u_{2}^{T}(x)\right]^{T} \in F_{2}$, then it can be observed that $z_{1}(x)=z_{2}(x)$, leading to $\left.\operatorname{Proj}_{\mathbb{R}^{d_{x}+1}} F_{1}=\operatorname{Proj}_{\mathbb{R}^{d_{x}+1}} F_{2}=F_{\left[x^{T}\right.}^{(i)}\right]^{T}$.

If $u_{1}(x) \neq u_{2}(x)$, then $F_{1}, F_{2}$ lie in a hyperplane of dimension $d_{x}+1$ which is orthogonal to the space of 
$\left[\begin{array}{ll}x^{T} z\end{array}\right]^{T}$. In this case, either $f_{p w a}(v)$ or $\widehat{f}(r)$ or both are not uniquely defined for some vertices $v \in \mathcal{V}\left(\mathcal{X}_{i}\right)$, some extreme rays $r \in \mathcal{R}\left(\mathcal{X}_{i}\right)$. This contradicts the construction in (6). Therefore, $F_{1}=F_{2}$ leading to the uniqueness of the optimal solution to (7).

Based on the above result, Algorithm 2 summarizes a procedure to solve the inverse optimality problem to recover a continuous PWA function defined over a convexly liftable cell complex of a polyhedron.

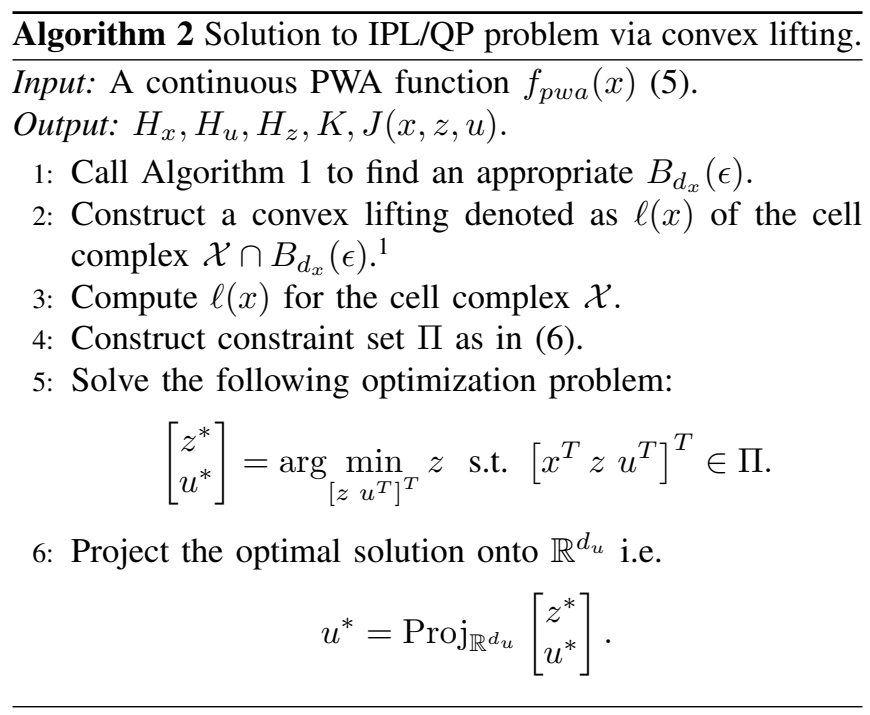

Note that this result can be trivially extended for removal of redundant constraints as shown in [12]. Similarly, extensions of inverse optimality with quadratic cost functions and discontinuous PWA functions are referred to [11].

\section{NUMERICAL EXAMPLES}

This section considers some numerical examples to illustrate the above results. All numerical examples in this paper are carried out in the environment of MPT 3.0 [8].

\section{Example 1}

This subsection considers a continuous PWA function defined over the whole space $\mathbb{R}$, as shown in Fig.1. This partition is the union of several segments:

$$
\begin{aligned}
\mathcal{X}_{1} & =\{x \in \mathbb{R} \mid x \leq-0.5\}, \\
\mathcal{X}_{i} & =\{x \in \mathbb{R} \mid 0.1(i-7) \leq x \leq 0.1(i-6)\}, i=2 \ldots 11, \\
\mathcal{X}_{12} & =\{x \in \mathbb{R} \mid x \geq 0.5\}
\end{aligned}
$$

A convex lifting of this partition is presented in Fig.2. A set of constraints for the formulation of inverse optimality problem, is shown in Fig.3 as the shaded pink polyhedron. It is shown therein that the line along the $x$-axis, represents the given partition $\mathcal{X}$, while the green curve represents the given PWA function associated with this partition. It can be observed that the solid pink line depicts the optimal solution to (7) whose image via the orthogonal projection onto $\mathbb{R}^{d_{u}}$ coincides with the given PWA function.

\footnotetext{
${ }^{1}$ An algorithm to construct a convex lifting of a bounded cell complex is presented in [15].
}

\section{Example 2}

The present subsection aims to illustrate the constructive solution with another continuous PWA function defined over a convexly liftable partition covering the whole space $\mathbb{R}^{2}$ as seen in Fig.4. This continuous PWA function is depicted in Fig.5. A convex lifting of the given partition can be found in Fig.6. Finally, the recovered PWA function is shown in Fig.7, and can be seen to be similar to the original one.

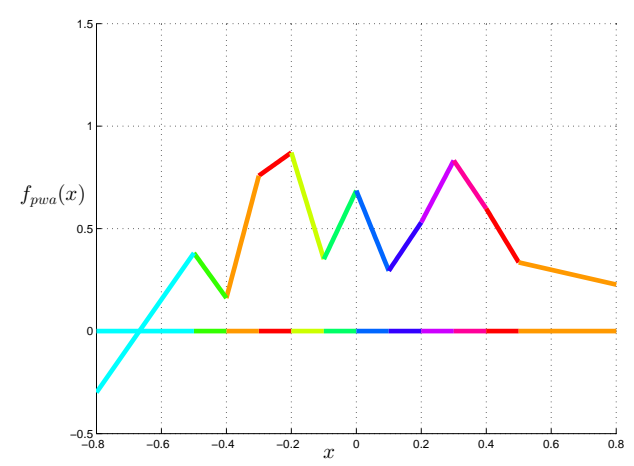

Fig. 1. Given PWA function defined over a partition of $\mathbb{R}$.

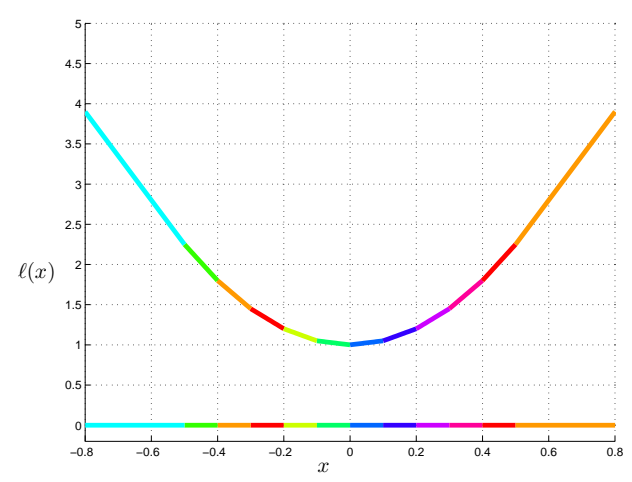

Fig. 2. A convex lifting of the partition in Fig.1.

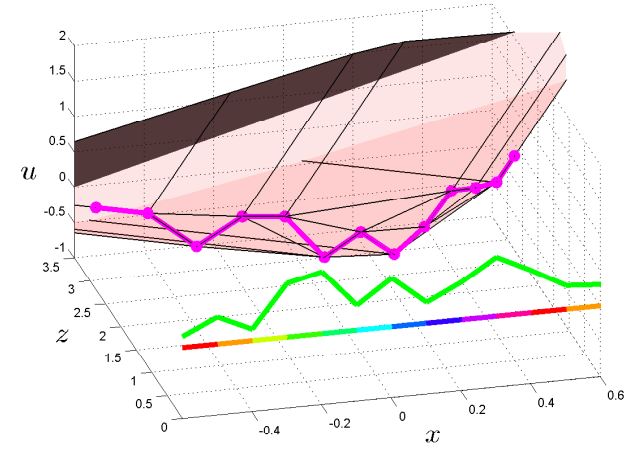

Fig. 3. An illustration of the constructive solution to inverse optimality problem in $\mathbb{R}^{3}$.

\section{CONCLUSIONS}

The present paper shows a procedure to recover a continuous PWA function defined over a polyhedral partition of 


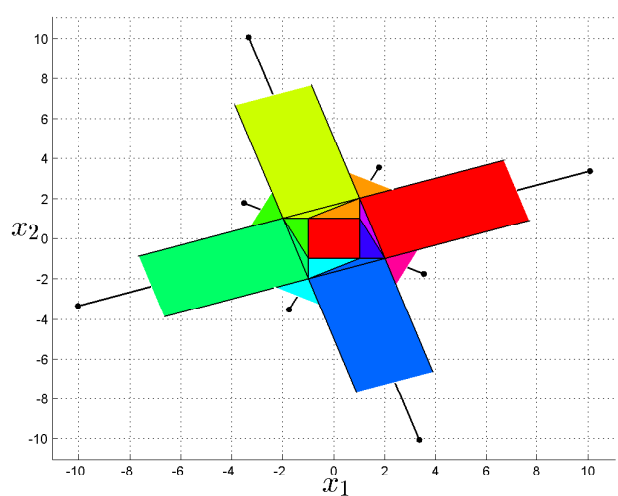

Fig. 4. A partition covering the whole space $\mathbb{R}^{2}$.

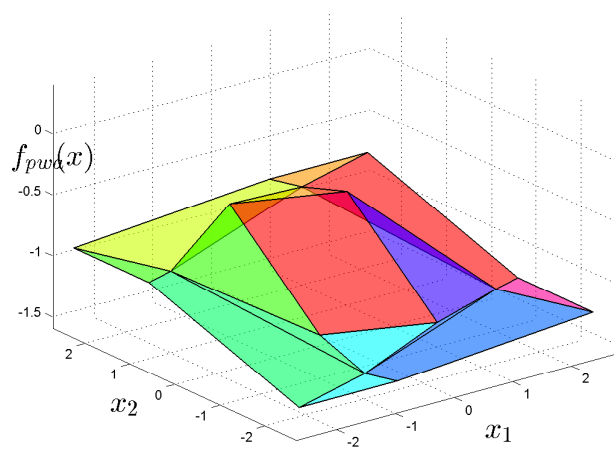

Fig. 5. A continuous PWA function associated with the partition in Fig.4.

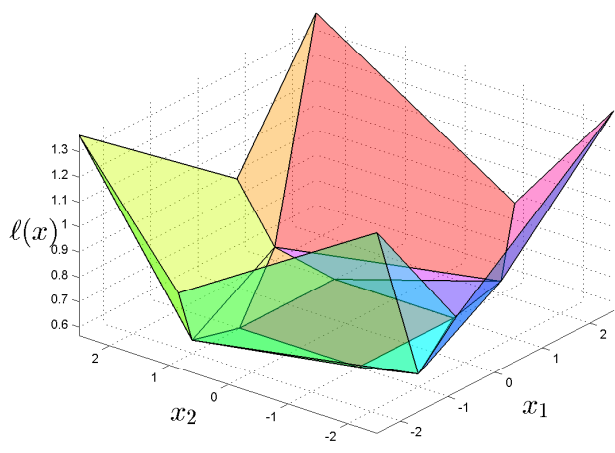

Fig. 6. A convex lifting of the partition in Fig.4.

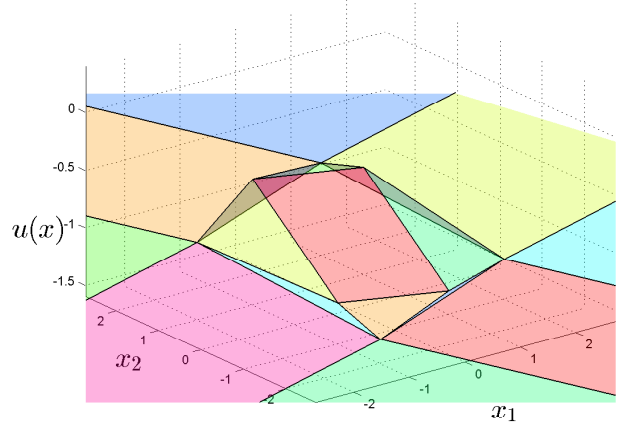

Fig. 7. The recovered PWA function. unbounded polyhedra. This solution is a simple extension of the constructive solution based on convex liftings put forward in previous results. Two numerical examples are presented to illustrate the constructive procedure proposed above.

\section{REFERENCES}

[1] M. Baes, M. Diehl, and I. Necoara, "Every continuous nonlinear control system can be obtained by parametric convex programming," IEEE tran on Automatic Control, vol. 53, no. 8, pp. 1963-1967, Sept 2008.

[2] A. Bemporad and C. Filippi, "An algorithm for approximate multiparametric convex programming," Computational optimization and applications, vol. 35, no. 1, pp. 87-108, 2006.

[3] A. Bemporad, M. Morari, V. Dua, and E. N. Pistikopoulos, "The explicit linear quadratic regulator for constrained systems," Automatica, vol. 38, no. 1, pp. 3-20, 2002.

[4] A. Grancharova and T. A. Johansen, Explicit Nonlinear Model Predictive Control. Springer, 2012.

[5] B. Grünbaum, Convex polytopes. Wiley Interscience, 1967.

[6] M. Gulan, N. Nguyen, S. Olaru, P. Rodriguez-Ayerbe, and B. Rohal'Ilkiv, "Implications of inverse parametric optimization in model predictive control." in Developments in Model-Based Optimization and Control, S. Olaru, A. Grancharova, and F. L. Pereira, Eds. Springer, 2015.

[7] A. B. Hempel, P. J. Goulart, and J. Lygeros, "Every continuous piecewise affine function can be obtained by solving a parametric linear program," in European Control Conference July 17-19, Zurich, Switzerland, 2013.

[8] M. Herceg, M. Kvasnica, C. Jones, and M. Morari, "MultiParametric Toolbox 3.0," in Proc. of the European Control Conference, Zürich, Switzerland, July 17-19 2013, pp. 502-510, http://control.ee.ethz.ch/ mpt.

[9] T. A. Johansen, "On multi-parametric nonlinear programming and explicit nonlinear model predictive control," in Decision and Control, Proceedings of the 41st IEEE Conference on, vol. 3, Dec 2002, pp. 2768-2773.

[10] - "Approximate explicit receding horizon control of constrained nonlinear systems," Automatica, vol. 40, no. 2, pp. 293 - 300, 2004.

[11] N. A. Nguyen, S. Olaru, and P. Rodriguez-Ayerbe, "Any discontinuous pwa function is optimal solution to a parametric linear programming problem," in 54th IEEE Conference on Decision and Control, Osaka, Japan, 2015.

[12] - "On the complexity of the convex liftings-based solution to inverse parametric convex programming problems," in European Control Conference, Linz, Austria, 2015.

[13] — "Recognition of additively weighted voronoi diagrams and weighted delaunay decompositions," in European Control Conference, Linz, Austria, 2015.

[14] N. A. Nguyen, S. Olaru, P. Rodriguez-Ayerbe, M. Hovd, and I. Necoara, "Inverse parametric convex programming problems via convex liftings," in 19th IFAC World Congress, Cape Town, South Africa, 2014.

[15] - "On the lifting problems and their connections with piecewise affine control law design," in European Control Conference, Strasbourg, France, 2014.

[16] S. Olaru and D. Dumur, "A parameterized polyhedra approach for explicit constrained predictive control," in Decision and Control, 43rd IEEE Conference on, vol. 2, 2004, pp. 1580-1585.

[17] — "On the continuity and complexity of control laws based on multiparametric linear programs," in Decision and Control, 45th Conference on. IEEE, 2006, pp. 5465-5470.

[18] E. N. Pistikopoulos, M. C. Georgiadis, and V. Dua, Multi-parametric programming. Wiley-vch, 2007.

[19] E. Pistikopoulos, "Perspectives in multiparametric programming and explicit model predictive control," AIChE journal, vol. 55, no. 8, pp. 1918-1925, 2009.

[20] K. Rybnikov, "Polyhedral partitions and stresses," Ph.D. dissertation, Queen University, Kingston, Ontario, Canada, 1999.

[21] A. Schrijver, Theory of linear and integer programming. John Wiley \& Sons, 1998.

[22] M. M. Seron, G. C. Goodwin, and J. A. Doná, "Characterisation of receding horizon control for constrained linear systems," Asian Journal of Control, vol. 5, no. 2, pp. 271-286, 2003. 